\title{
TOPOLOGY OPTIMIZATION OF PLASTIC PARTS FOR INJECTION MOLDING
}

\author{
Kathryn Oliver, Sohel Anwar, and Andres Tovar ${ }^{1}$ \\ Department of Mechanical and Energy Engineering \\ Purdue School of Engineering and Technology \\ Indiana University-Purdue University Indianapolis \\ Indianapolis, Indiana, USA
}

\begin{abstract}
Topology optimization is broadly recognized as a design approach to generate high-performance conceptual designs suitable for freeform fabrication, e.g., additive manufacturing. When other fabrication methods are considered, topology optimization must integrate manufacturing constraints. The integration of constraints for extrusion and casting has been addressed in the past by a few researcher groups. In this work, extrusion and casting constraints are revisited and extended to include plastic injection. The proposed method relies on the use of intersection planes and the definition of a parting line within the planes. The resulting topologies can be injected in a two-plate mold without the use of inserts. The implementation and results of the proposed approach are demonstrated in classic threedimensional problems that include a cantilevered beam with different load conditions.
\end{abstract}

Keywords: Structural Optimization; Finite Element Analysis; Plastic Injection; Manufacturing Constraints

\section{INTRODUCTION}

The manufacturability of a design greatly impacts the cost of production. Injection molding is a cost-effective manufacturing process for intricate parts created in large quantities. To minimize production costs, various design factors should be taken into consideration including interior holes and mold parting lines. [1] In this work, topology optimization techniques are used in a three-dimensional space to create optimal designs which conform to specific injection molding manufacturing constraints. The typical structure of designs resulting from standard topology optimization problems are difficult and expensive to manufacture without modifications. The complex structures may be optimal for performance and mass constraints, but they do not readily conform to inexpensive production processes. Until the early 1990s, structural optimization was only used to improve upon current designs, not necessarily to develop initial designs [2].

Topology optimization is an iterative method to determine the optimal material distribution within a design space for a defined loading condition. A common objective is to minimize compliance while also achieving a specified volume fraction and, in turn, a specific weight. The adjustable design variables in these density-based problems are the normalized element densities. A density of zero implies that there is a void, or no material in that space while a density of one implies the element is fully solid. Using topology optimization for initial concept design can lead to innovative designs with high performance attributes. Beginning with Bendsøe and Kikuchi's seminal paper in 1988 [3] topology optimization has evolved significantly.

Current topology optimization techniques create complex structures, which require several post-processing iterations to create a manufacturable product. Manufacturing constraints need to be applied based on the type of manufacturing. Patel et al. developed a method using topology optimization and cellular automaton to generate fixed cross-sectional structures suitable for extrusion manufacturing [4]. Vatanabe et al. applied a unified projection-based approach to apply various manufacturing constraints, including minimum member size, symmetry, and minimum hole size. This method creates designs for multiple manufacturing processes including casting, milling, turning, extrusion and rolling [5].

Topology optimization has also been improved for additive manufacturing. A main difference between injection molding and additive manufacturing is that the parts are built upright, layer by layer, necessitating support structures in most cases.

'Contact author: tovara@iupui.edu

This is the author's manuscript of the article published in final edited form as: 
These supports increase the material usage and production time which Ranjan et al. aim to minimize in their topology optimization techniques [6]. Furthering this work, Mhaspsekar et al. refine the process for minimizing the volume of support structures as well as minimizing the number of thin features [7]. Current research has been performed in optimizing the process parameters in injection molding machinery such as melt temperature, mold temperature, injection time and injection pressure. [8] However, these optimizations are for specific already designed products.

The focus of this paper is the initial design phase of injection molded products and to explore the procedures necessary to ease manufacturing using injection molding. To create designs which satisfy manufacturing constraints, each iteration of the topology optimization analysis is updated. The output design of each iteration is altered to meet specified manufacturing requirements all conducive to injection molding, including extrusion, open mold casting and two plate molds considering a parting plane. The modified design becomes the input of the next iteration of the topology optimization analysis. This process is repeated until the modified designs converge.

\section{MATERIALS AND METHODS}

When assessing the topology optimization function outputs, multiple manufacturing adjustments would result in final designs suitable for injection molding. In this paper, extrusion, open mold casting and two plate injection molding processes were analyzed. Extrusion and casting techniques were taken from literature and are explained with slight modifications. The new constraint analyzed in this work is two plate injection molding using the context of a parting line. An efficient topology optimization tool is the top3d code by Liu and Tovar [9]. The top $3 \mathrm{~d}$ code is modified and utilized in this work.

\subsection{Extrusion Process}

In an extruded part, the design needs a uniform cross section. If the design is finite, it is also viable for injection molding. The design process for extrusion consists of two steps: projection and extrusion.

Projection: To ensure a uniform cross section, the output design from topology optimization in the design domain $\Omega$ is projected onto a plane $\Omega^{\prime}$ perpendicular to the extrusion direction as seen in Figure 1.

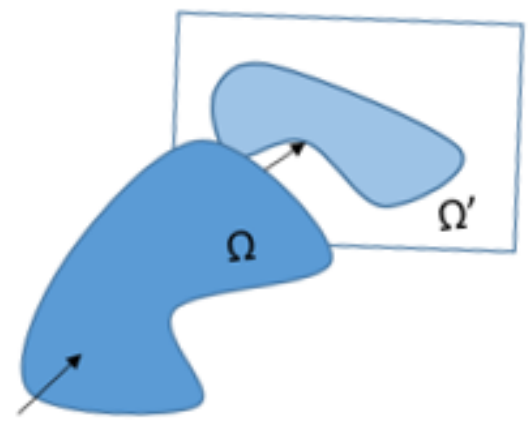

Figure 1: Projection to a plane perpendicular to the extrusion direction.

Extrusion: For the next step of the topology optimization process, the projected design on $\Omega^{\prime}$ is extruded to the original width of the design space as seen in Figure 2. The extruded design is the input of the next iteration of the topology optimization analysis. This process of projection and extrusion is repeated until convergence, i.e., no changes in the extruded design.

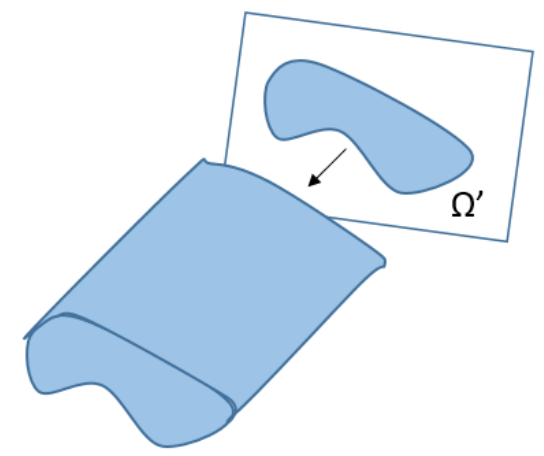

Figure 2: Extrusion from the projection plane.

\subsection{Open Mold Casting Process}

Open mold casting utilizes a single sided mold for simple and low-cost production. In this scenario, the output of the topology optimization function is analyzed in direction of the mold. For open mold casting, the design approach consists again of two steps: intersection and rasterization.

Intersection: Cutting planes are made perpendicular to the mold face all along the design space. For each cutting plane, the intersected design space $\Omega^{\prime}{ }_{j}$ is analyzed. 

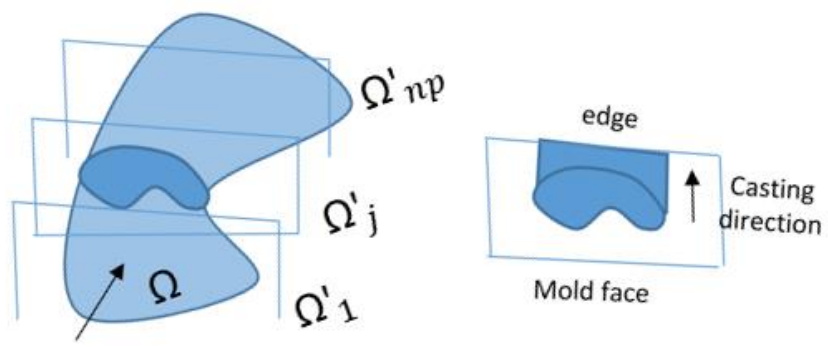

Figure 3: Open mold casting process: intersection from cutting planes and rasterization in the casting direction

Rasterization: If the normalized density is higher than a defined threshold value, the cutting plane is rasterized or filled from that element towards the open edge of the mold. A visual for this process can be seen in Figure 3.

\subsection{Parting Plane Process}

To alter the iterations for the topology optimization problem, parting planes were taken into consideration for a twoplate mold. A parting line can be placed in the $x, y$, or $z$ plane to demonstrate the meeting point of a two-plate mold. This twoplate mold process utilizes a similar fill operation as open mold casting to update the input geometry for the topology optimization problem. After creating the cutting planes all along the design space, a rasterization or fill density is applied between the element that meets the density threshold and the parting plane. This means that the design is evaluated from two different directions meeting at the parting plane in between. The result of this modification is a solid part between the two mold faces. The process is visualized in Figure 4.

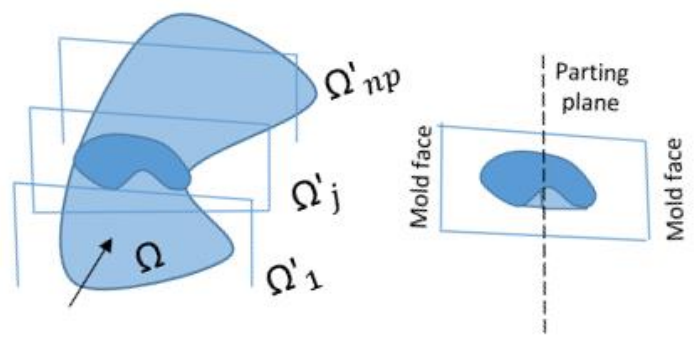

Figure 4: Two plate parting line process.

\section{RESULTS AND DISCUSSION}

In this work, three different processes were used all resulting in designs which are manufacturable using injection molding. The three processes include applying extrusion, open mold casting, and parting line adjustments to each iteration of the topology optimization analysis. These processes are displayed through two loading conditions for a cantilever with a distributed load and a single load.

\subsection{Extrusion Implementation}

To update the topology optimization problem, the initial design is projected using the average densities in the extrusion direction. The extrusion projections the first iteration of a simple cantilever beam with a distributed load in the $\mathrm{x}, \mathrm{y}$ and $\mathrm{z}$ extrusion directions can be seen in Figure 5.

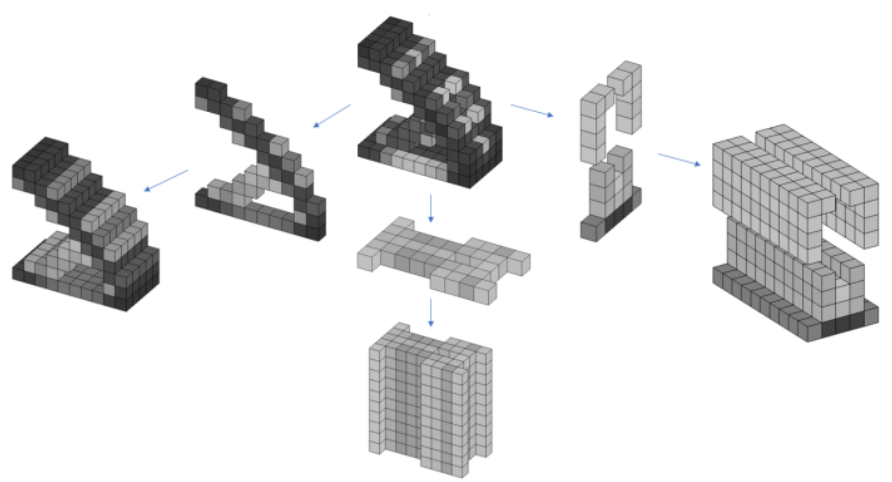

Figure 5: Extrusion of a design in various projection planes using the mean density

When the iterations converge to a single result, the optimal designs in each extrusion direction can be evaluated to find the design with mass and compliance values suitable to the designer. The designs resulting from different extrusion directions are not necessarily always feasible. In Figure 5 the result on the left is not a feasible design since not all of the elements are touching. The potential for this result is mitigated using the maximum density for the projection as seen in Figure 6. However, these designs will have a larger volume fraction than the target and can result in the entire design space filled.

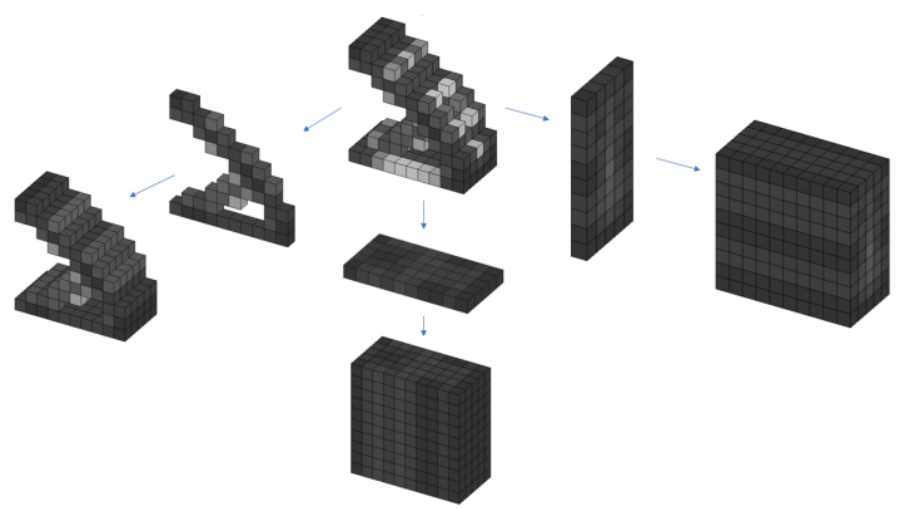

Figure 6: Extrusion of a design in various projection planes using maximum density. 


\subsection{Open Mold Casting Implementation}

The initial topology optimization design is adjusted for open mold casting in 4 different planes. The intention is that in the next iteration of topology optimization, an open molding feasible design will be the input. Figure 7 displays the various possibilities for altering the same simple cantilever beam from section 3.2 .

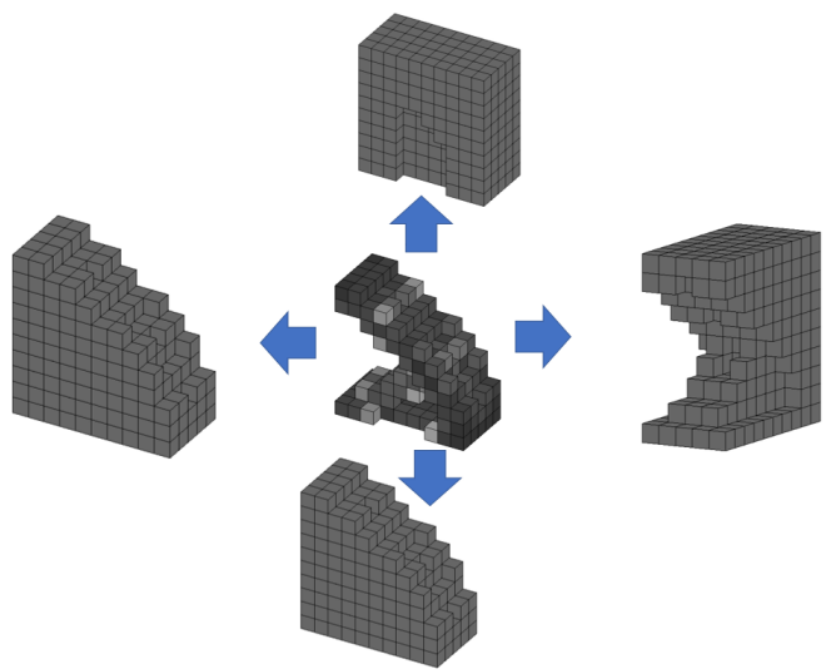

Figure 7: Open mold casting modification on a topology optimization iteration.

Similar to the extrusion case, the optimal designs in various directions can be evaluated for the case with the best performance and mass characteristics.

\subsection{Parting Line Implementation}

When applied to a three-dimensional part, a twodimensional plane is chosen for the place where the two mold plates meet. The material is analyzed for each element then back filled until that parting plane. Figure 8 displays how adding a parting line in the middle of the cantilever example creates a combination of the two corresponding open mold designs. These designs can evaluated using the same technique discussed in sections 3.2 and 3.3 to determine the optimal parting plane location.

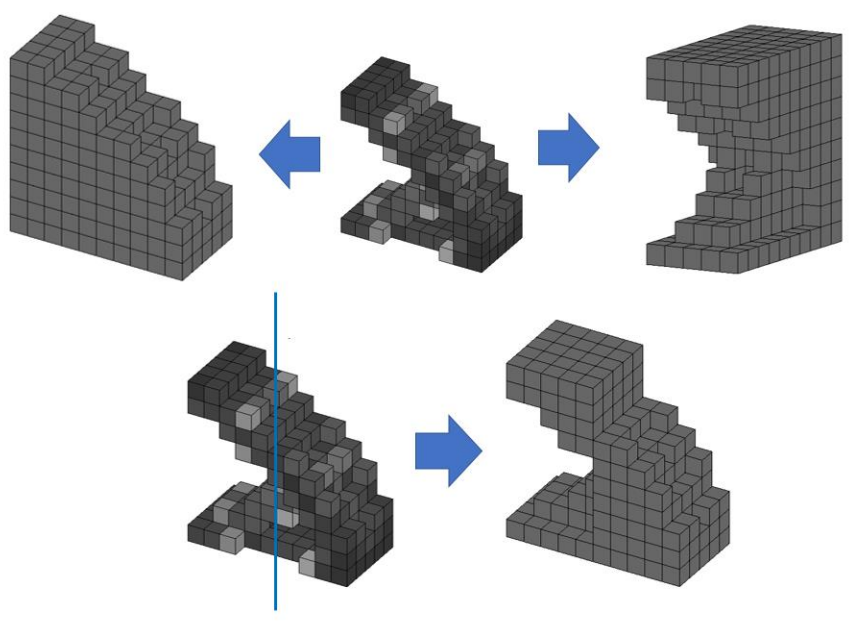

Figure 8: Cantilever example of relationship between open mold casting and parting line modifications.

\subsection{Cantilever with Distributed Load Example}

In the first example, the design space was set up $60 \times 6 \times 6$ with a total of 2,160 elements. The load cases and supports can be seen in Figure 9 below, applying a distributed load to the edge of the beam.

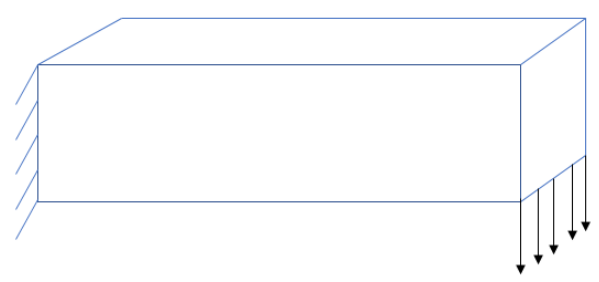

Figure 9: Loading conditions for the distributed load cantilever example problem.

First, the extrusion alterations were made to ensure a uniform cross section as outlined in section 2.2. The topology optimization program ran for a maximum of ten iterations, stopping the loop if the minimum change between the normalized projection densities was less than 0.01 . Table 1 displays the results of the optimization for parts extruded in the $x, y$ and $z$ planes. 
Table 1: Design for extrusion of a cantilevered beam with a distributed load

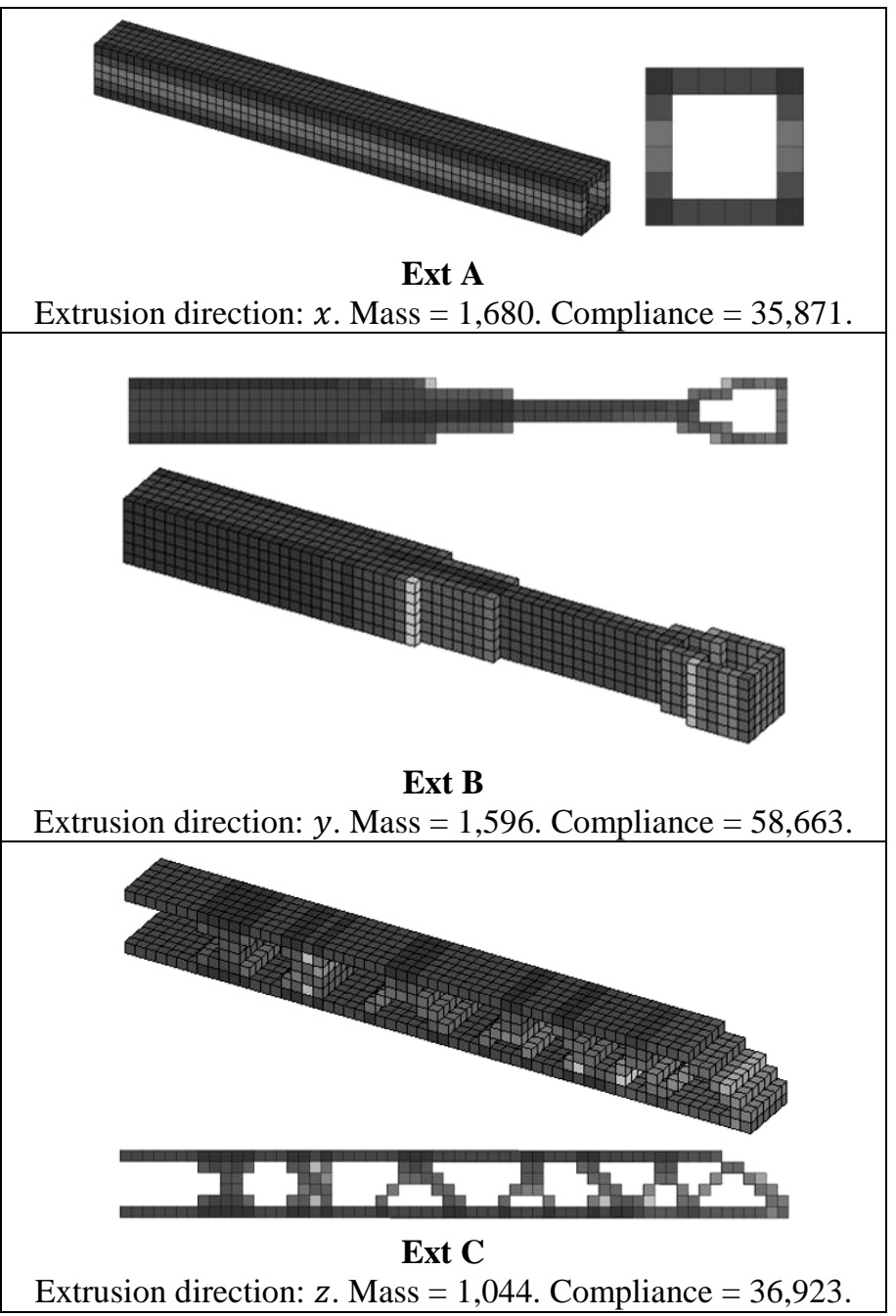

The topology optimization analysis was repeated utilizing the process for implementing a parting plane in the design space. For simplicity the parting line was placed in the center of the part in each plane but could be placed elsewhere. These results are shown in Table 2. The open mold casting process could be achieved by moving the parting line to the edge of the design space.
Table 2: Design for injection of a cantilevered beam with a distributed load

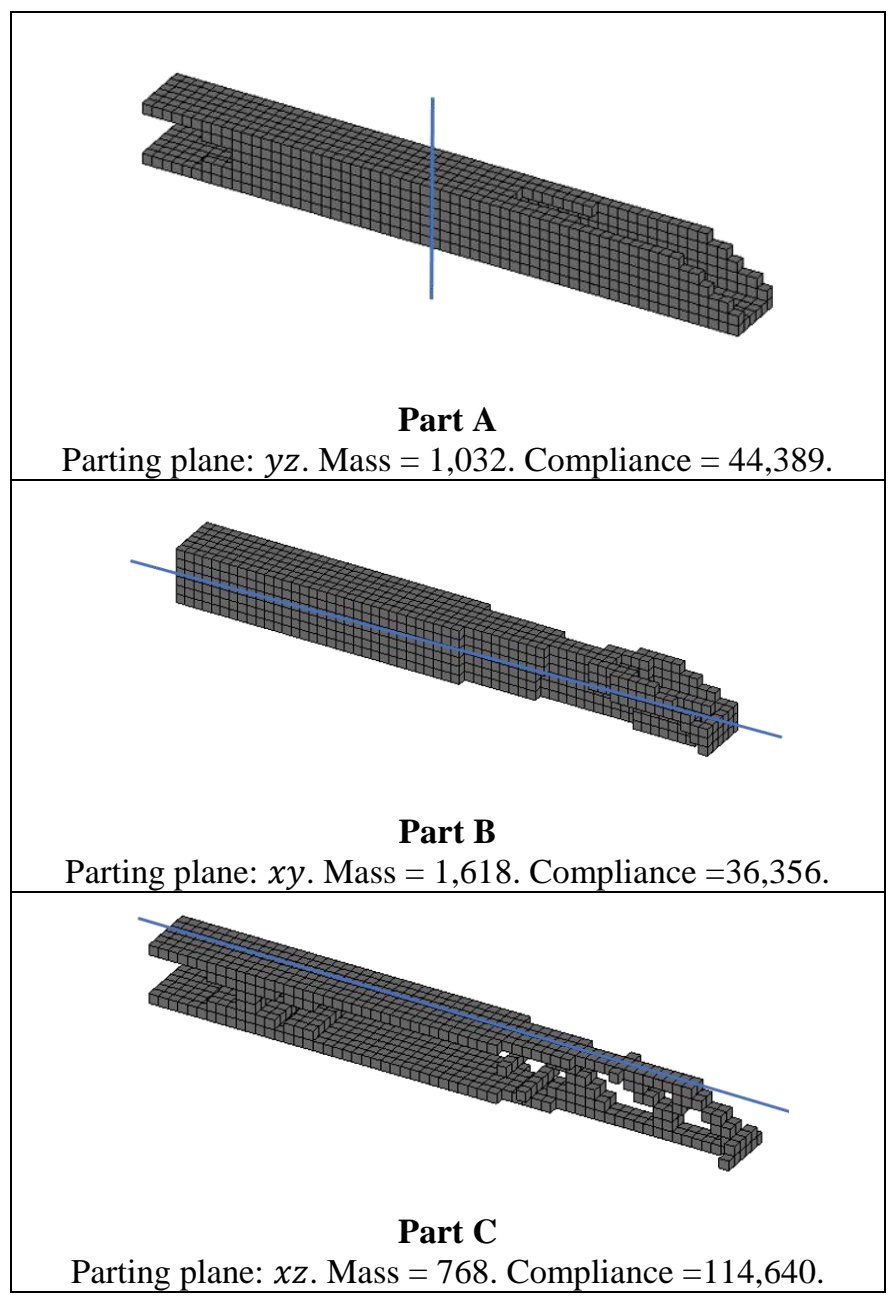

To analyze these results, we have the mass using a density equal to one and compliance. Ideally, both mass and compliance would be minimized. The best design depends on preferences between the two. To visualize the results Figure 10 below shows the mass vs compliance results for each design. 


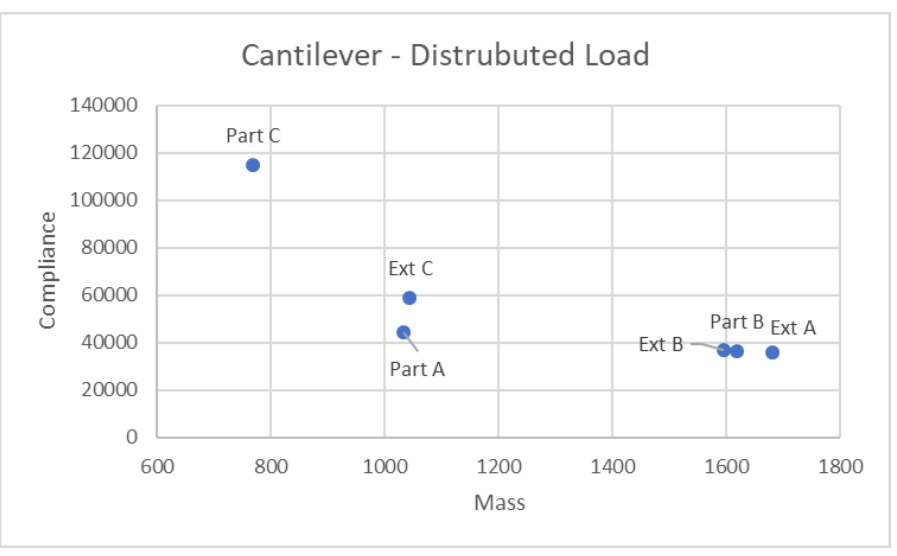

Figure 10: Mass vs compliance of resulting designs of distributed load cantilever.

\subsection{Cantilever beam with Single Load Example}

Using the same design space as the distributed load cantilever example, the same process was repeated using the loading conditions in Figure 11.

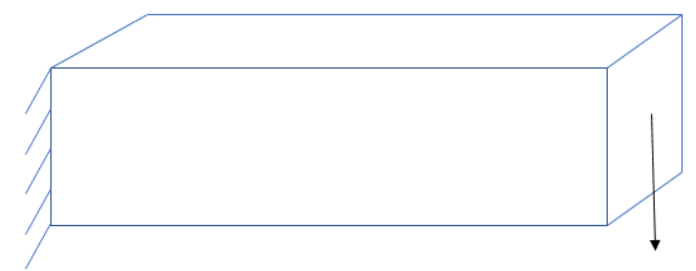

Figure 11: Loading conditions for a single load example problem.

Table 3 displays the final extrusion-based designs for the extrusions in the $x, y$ and $z$ directions.
Table 3: Design for extrusion of a cantilevered beam with a single load

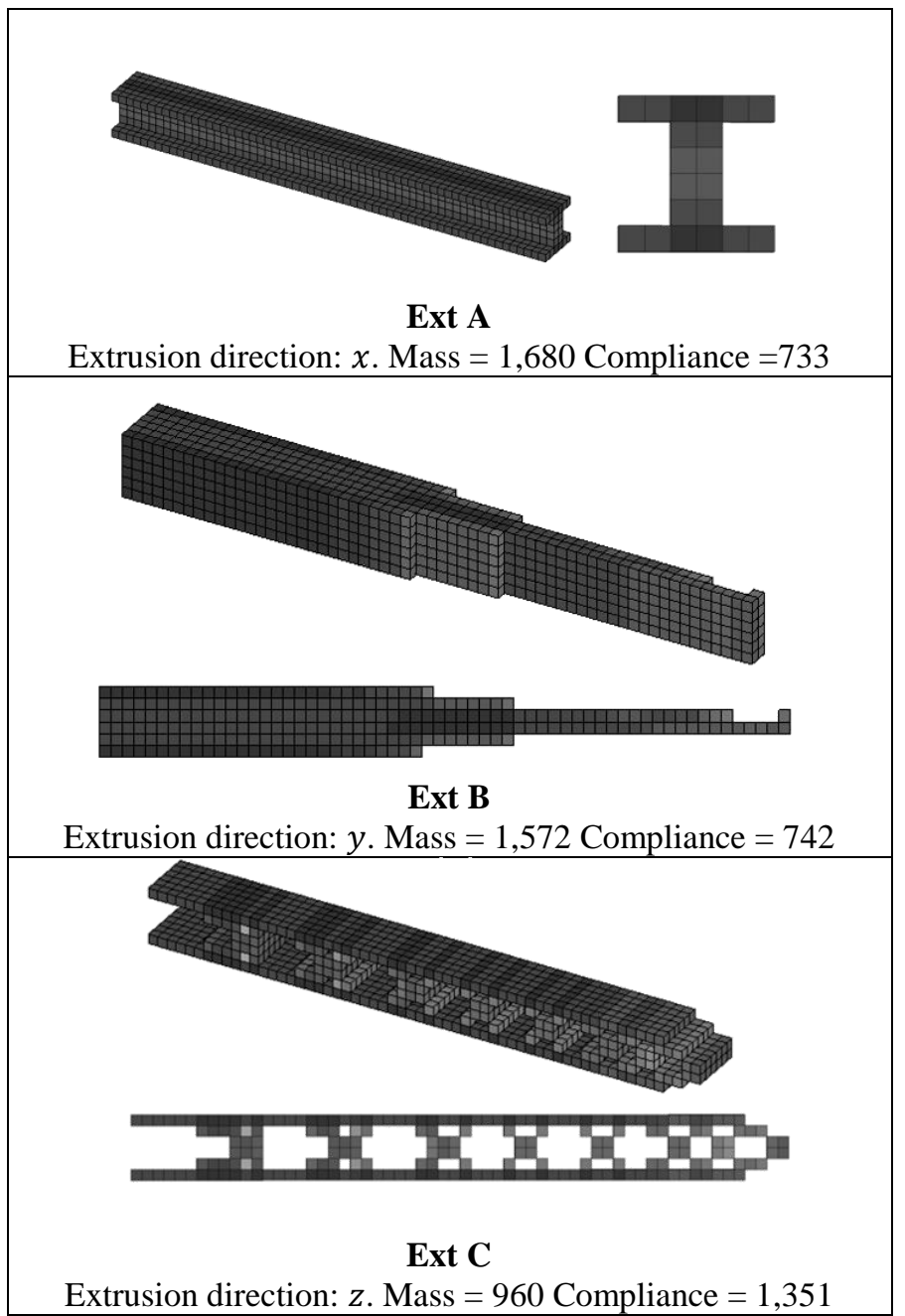

Using the same procedure as in the distributed load cantilever example, the iterations of the topology optimization are updated using the parting planes process outlined in section 2.4 . 
Table 4: Design for injection of a cantilevered beam with a single load

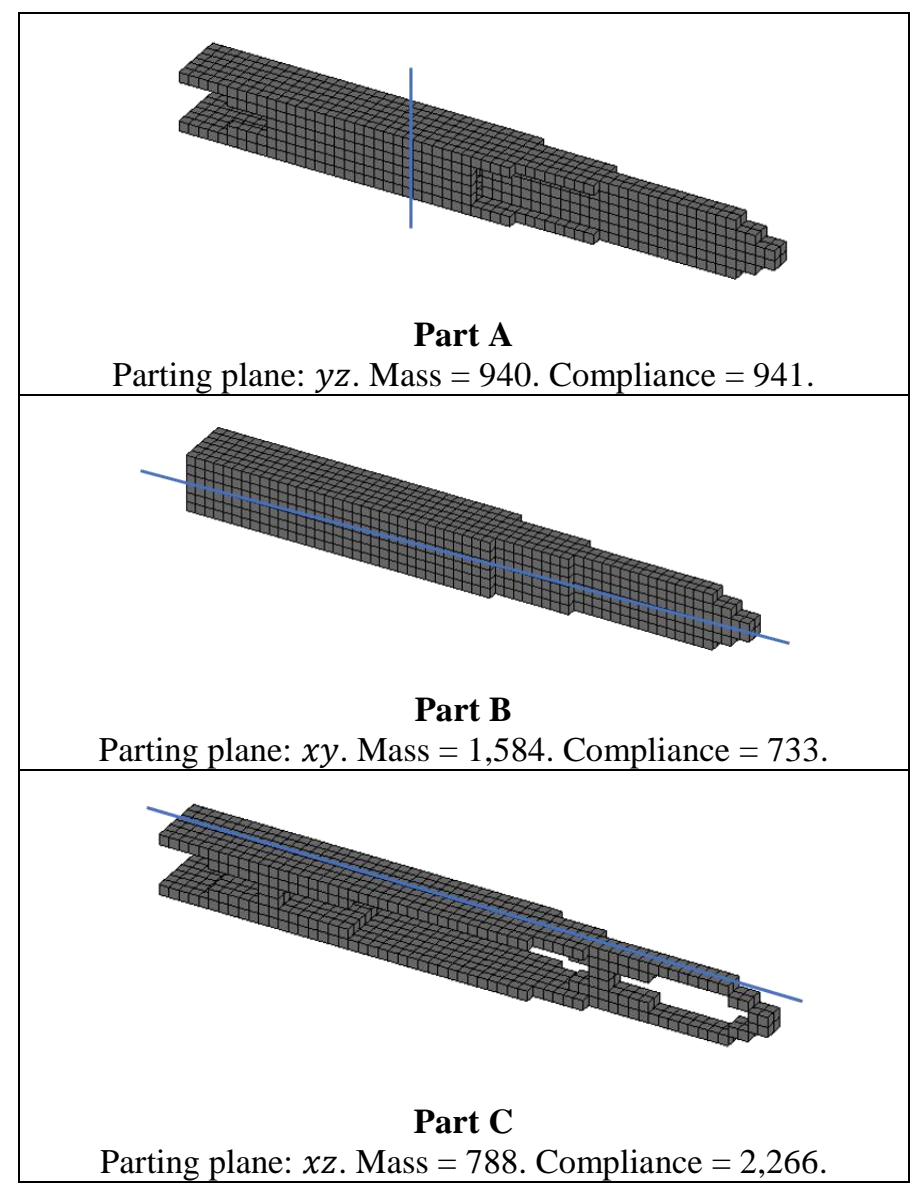

The results in Table 3 and Table 4 also show the mass using a density of one and compliance. These factors should be taken into account when choosing a design. Figure 12 shows their relationship for each design.

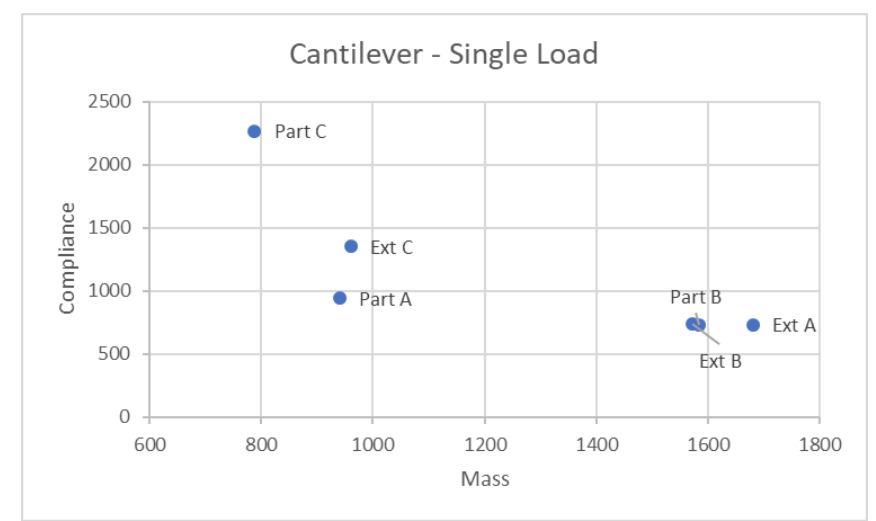

Figure 12: Mass vs compliance graph of resulting designs of single load cantilever.

\section{CONCLUSION}

Topology optimization is a great tool for maximizing stiffness while limiting the material used in the designs, however, the standard results do not conform with manufacturing guidelines. This work illustrates various constraints applicable to topology optimization to improve the manufacturability of the design including implementing a parting line. Limitations of this work include determining a threshold density value to use in the various modifications of the topology optimization inputs, rotating cutting and projection planes to find more unconventional design structures and strategies for evaluating the final results.

\section{ACKNOWLEDGEMENTS}

This work was made possible by the support from the Department of Mechanical and Energy Engineering, IUPUI and by the National Science Foundation NRT-IGE grant number 1633426.

\section{REFERENCES}

[1] J. G. Bralla, Handbook of Product Design for Manufacturing, Jersey City, New Jersey: McGraw-Hill Book Company, 1986.

[2] U. Schramm and M. Zhou, "Recent Developments in the Commercial Implementation of Topology Optimization," in IUTAM Symposium on Topological Design Optimization of Structures, Machines and Materials: Status and Perspectives, 2006.

[3] M. P. Bendsoe and Kikuchi, "Generating Optimal Topologies in Structural Design Using a Homogenization Method," Computer Methods in Applied Mechanics and Engineering, vol. 71, pp. 197-224, 1988.

[4] N. M. Patel, C. L. Peninger and J. E. Renaud, "Topology Synthesis of Extrusion-Based Nonlinear Transient Designs," Journal of Mechanical Design, vol. 131, pp. 061003-1- 061003-11, 2009.

[5] S. L. Vatanabe, T. N. Lippi, C. R. de Lima, G. H. Paulino and E. C. Silva, "Topology Optimization with Manufacturing Constraints: A Unified Projection-based Approach," Advances in Engineering Software, vol. 100, pp. 97-112, 2016.

[6] R. Ranjan, R. Samant and S. Anand, "Integration of Design for Manufacturing Methods With Topology Optimization in Additive Manufacturing," Journal of Manufacturing Science and Engineering, vol. 139, pp. 061007-1 - 06100713, 2017.

[7] K. Mhapsekar, M. McConaha and S. Anand, "Additive Manufacturing Constriants in Topology Optimization for Improved Manufacturability," Journal of Manufacturing Science and Engineering, vol. 140, pp. 051017-1 - 051017$16,2018$. 
[8] H. Gao, Y. Zhang, Y. Fu, T. Mao, H. Zhou and D. Li, "Process Parameters Optimization Using a Novel Classification Model for Plastic Injection Molding," Int J Adv Manufacturing Technology, vol. 94, pp. 357-370, 2018.

[9] K. Liu and A. Tovar, "An Effiecient 3D Topology Optimization Code Written in Matlab," Structural and Multidisciplanry Optimization, vol. 50, no. 6, pp. 11751196, 2014. 